\title{
MHC class II genotype- and MHC class I and II phenotype- related parameters in sporadic colorectal cancer
}

\author{
TORALF WOLKERSDÖRFER ${ }^{1}$, MONIKA FÜSSEL ${ }^{2}$, TOBIAS KIESLICH ${ }^{3}$, DANIEL NEUREITER ${ }^{4}$, \\ FRIEDER BERR $^{3}$, DANIELA AUST $^{5}$ and GERNOT W. WOLKERSDÖRFER ${ }^{3}$ \\ ${ }^{1}$ Department of Surgery, Georgius Agricola Hospital, Saalfeld; ${ }^{2}$ MMD Molecular Diagnostics GmbH, Dresden, \\ Germany; ${ }^{3}$ Department of Internal Medicine I, ${ }^{4}$ Institute of Pathology, Paracelsus Medical University, \\ Salzburg, Austria; ${ }^{5}$ Department of Pathology, Technical University of Dresden, Dresden, Germany
}

Received May 6, 2011; Accepted July 1, 2011

DOI: $10.3892 / o r .2011 .1427$

\begin{abstract}
The underlying etiological cause of non-hereditary colorectal cancer has yet to be determined. The adenomacarcinoma sequence is widely accepted, however, a sole trigger has not been specified. Therefore, we sought to further define genotypic and phenotypic parameters that could be involved in promoting a possible infection, inflammation and hyperproliferation, followed by the adenoma-carcinoma sequence. Expression of phenotype-related parameters for MHC class I (HLA-A N-20 and $\beta 2$ microglobulin) and class II (HLA-DR $\alpha$ and HLA-DR) as well as CD45 and carcinoembryonic antigen (CEA) were investigated immunohistochemically in a series of 93 colorectal cancers. Additionally, in 49 of the tumours the MHC class II genotype was analysed. MHC class II genotype analyses revealed a tendency towards DRB1 ${ }^{*} 08$ and DQB1*04. A significant association among the MHC class I markers or the MHC class II markers was found. No difference in marker expression could be detected between tumour and stromal tissue, however a significant inverse expression existed for markers of the functionally different class I or II systems. With the exception of CEA, there was no correlation between expression of any marker and tumour grade. Only $2 \%$ of tumours expressed no markers for MHC class I and II. Further studies on MHC class I and II genotype and phenotype relation in colorectal cancer may help to identify trigger mechanisms for tumourigenesis, involved markers and possible mechanisms of subsequent immune escape.
\end{abstract}

\section{Introduction}

The adenoma-carcinoma sequence has been widely accepted in the development of sporadic colorectal carcinoma (1). In

Correspondence to: Dr Gernot W. Wolkersdörfer, Department of Internal Medicine I, Paracelsus Medical University, Müllner Hauptstrasse 48, Salzburg 5020, Austria

E-mail: g.wolkersdoerfer@salk.at

Key words: tumour, stroma, microenvironment, major histocompatibility complex, human leucocyte antigen, expression addition, low adiponectin levels and high insulin levels have recently been linked to a higher incidence of colorectal carcinoma and may, therefore, provide the metabolic background for this sequence $(2,3)$. However, the initial trigger has yet to be defined.

Inflammation of the intestinal mucosa could be part of such a triggering mechanism. Indeed, inflammatory bowel diseases have a higher incidence of colorectal cancer and, therefore, more strict regimens of surveillance have been incorporated in recent guidelines (4-6). A genetic predisposition in chronic inflammatory bowel diseases has been found and linked to genes related to inflammation and the immune response (7). Inflammation and immune response involve molecules of antigen processing, presentation and cellular and cytokine mediated interaction with or among cells of the immune system (8). As such, among major risk factors in Crohn's disease are mutations in NOD2 and Atg16L1, both resulting in defective macroautophagy and subsequent MHC class II presentation with ineffective CD4 T-cell response $(9,10)$. Indeed, the expression of inflammatory genes including MHC class II genes even in sporadic adenocarcinoma has been found to be associated with poor prognosis (11).

The MHC system plays a key role in antigen processing, presentation and cellular interaction. Even an MHC restriction to certain genotypes has been suggested in tumours like melanoma, tumours of the stomach, the liver, the prostate, the ovary, thyroid gland, the lungs, but also some types of leukaemia or lymphoma (12-35). However, the link to the MHC system is not unequivocal in these tumours. Contrary to that, the prevalence of DQB1 and DRB1*03 has been proven to cause an altered response to the human papillomavirus in the cervix. Here, these MHC class II types allow for an infection with HPV 16, 18, 31 or 33, which leads to cellular overexpression of viral oncogenes such as E5, E6 and E7. As a result, PDGF-receptors will be constitutively active, the p53-tumour suppressor gene product is inactivated by ubiquitination and binding to $\mathrm{pRB}$ with subsequent release of E2F-1 and S-phase progression, all together processes, that allow for the development of tumour $(20,36,37)$.

The link between the MHC system and tumour formation in sporadic colorectal cancers has up to now not been conclusively explained. Older data could not demonstrate a link between MHC expression and colorectal cancer (38). On 
the other hand, even a prognostic relevance has been suggested when MHC class I molecules have been down-regulated in rectal cancer, although such data is not unequivocal (39). HLA-A, -B, - C expression in tumours was found to be similar to normal surrounding tissue, whilst a deviation from this similarity was again correlated with poor prognosis (40). Poor differentiation was found to be associated with a loss of MHC class I expression (41) but with expression of MHC class II genes (42). Therefore, we sought to further analyse tumour samples from sporadic colorectal carcinoma with respect to their MHC class II genotype- and MHC class I and II phenotype-related parameters.

\section{Materials and methods}

Patients and tissues samples. Two hundred and fifty-eight tissue samples from 93 patients with colorectal carcinoma surgically removed between 1997 and 2000, were included and examined in this study (88 adenocarcinoma, 5 mucinous carcinoma). The study was approved by the Ethics Committee of the University of Technology, Dresden, Germany, and conducted after written informed consent was obtained from the patients. The tumours were analysed according to the grade, differentiation, histological type and according to the TNM classification; tumour samples were collected from each tumour, fixed in $10 \%$ formalin, $\mathrm{pH} 7.4$, and embedded in paraffin. Slices of $6 \mu \mathrm{m}$ were used in all procedures.

Immunohistochemistry. Paraffin sections were separately immunostained for carcinoembryonic antigen (CEA, clone 11-7, Dako, Hamburg, Germany), CD45 (clones 2B11 and PD7/26, Dako),HLA-DR $\alpha$ chain (TAL.1B5, Dako), HLA-DR (clone 3/43, Dako), HLA-A (N-20, sc-17653; Santa Cruz Biotechnology, Inc., Heidelberg, Germany), $\beta 2$ microglobulin (BBM.1, sc-13565; Santa Cruz Biotechnology). As a control, the specific antiserum was replaced by an isotype-immune mouse $\mathrm{IgG}_{1}, \kappa$ (anti-TNP) serum (Pharmingen, Hamburg, Germany).

The reaction was visualised using the avidin-biotin staining method according to the catalysed signal amplification (CSA) system with AEC or DAB chromogen (Immunotech, Hamburg, Germany). Slides were counterstained with haematoxylin, rinsed in water and mounted in glycerol gelatine (SigmaAldrich, Munich, Germany).

Staining was than evaluated by counting stained cells per 100 and grouping into $0 ; 1,1-5 ; 2,6-25 ; 3,26-50 ; 4,51-75$; $5,76-100$ cells and estimating the intensity of staining (from 0 missing to 3 very strong). The product of both (ratio and intensity) has been referred to as the SI (staining index) and was used for further analyses. CD 45 staining was evaluated by counting stained cells per 100 and grouping into $0 ; 1,1-10 ; 2$, $11-50,3,>50$ cells without estimating the intensity of staining. Evaluation was conducted for tumourous and stromal tissue separately.

DNA extraction. DNA extraction was carried out as previously described (43). In brief, deparaffinised sections were air-dried and incubated in $1 \mathrm{M}$ guanidium thiocyanate overnight at room temperature, washed in TE and proteinase K-digested for $72 \mathrm{~h}$ at $55^{\circ} \mathrm{C}$. After centrifugation the supernatant was phenol and chloroform extracted.
Table I. Characteristics of tumours and patients.

Characteristics

Gender, n (\%)
Male
Female

Female $38(40.9)$

Age average (years)

Male

66.2

Female

Tumour grade, $\%$

II

III

IV

T stage, $\%$

$1 \quad 3.7$

$2-13.8$

$3 \quad 56.1$

$4 \quad 11.0$

Metastatic disease, $\%$

Distant $\quad 13.9$

Lymph nodes $\quad 40.8$

Lymph nodes in T1 0.0

Lymph nodes in T2 2.2

Location, \%

Cecum

Ascending colon

18.3

Transverse colon $\quad 5.3$

Descending colon $\quad 4.3$

Sigmoid colon $\quad 35.5$

Rectum

29.0

HLA genotype determination. Low resolution HLA-genotyping of genomic DNA was carried out using the Amplicor HLA DRB test according to the suppliers' instructions (Dynal, Hamburg, Germany) for the DRB1* and DQB1* loci.

Statistical analyses. Statistical analyses were performed with SPSS software using the $Z_{\max }$ test (46) for comparison of MHC class II DR and DQ locus genotypes in carcinoma. HLA gene frequencies of normal bone marrow donors of the German population have been published by Mueller et al (44). They were calculated to be 0.1073 for DRB1*01, 0.0159 for DRB1*02 and 0.1007 for DRB1*03. The frequencies were used to generate $Z$-values for the different alleles. Results were considered statistically significant if $\mathrm{P}<0.05$.

Statistical analyses of HLA phenotype-related parameters were performed with the Prism GraphPad software (GraphPad Software Inc., La Jolla, CA, USA) using the two-tailed unpaired t-test or the F-test of variances to evaluate tumour characteristics and immunohistochemistry data. Results were considered statistically significant if $\mathrm{P}<0.05$. Evaluations were carried out to compare tumourous and stromal tissue. Furthermore, tissues were grouped according to low and high 

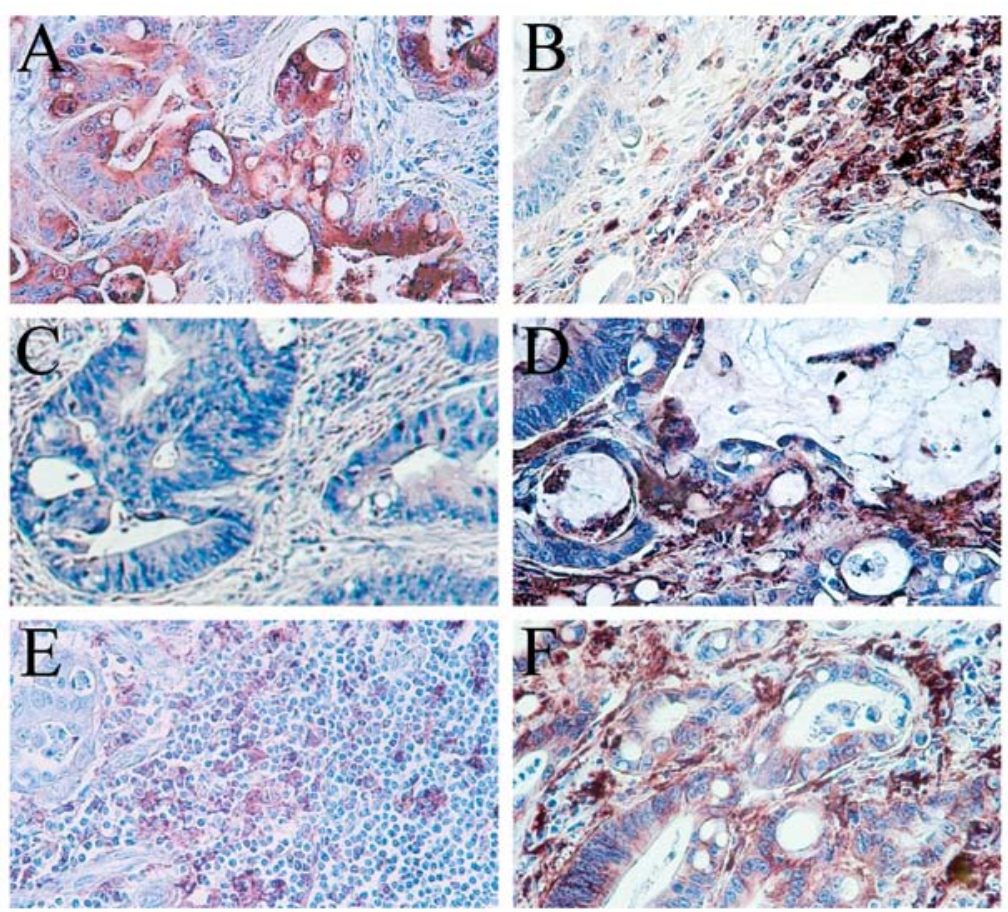

Figure 1. Typical staining patterns of a colorectal carcinoma. Specimens were stained against (A) CEA, (B) CD45, (C) HLA-A, (D) $\beta 2$ microglobulin, (E) HLA-DR $\alpha$ and (F) HLA-DR.

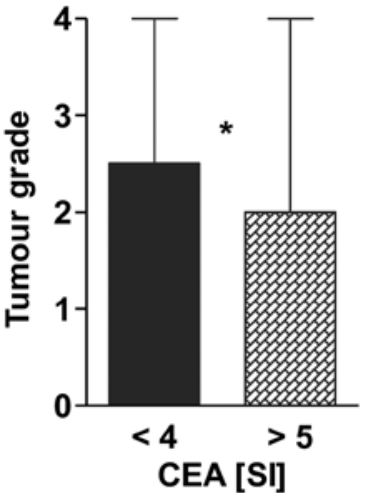

Figure 2. Comparison of tumours with lower and higher SI for CEA. The lower differentiation in tumours with a higher tumour grade correlated with a lower expression of CEA. "Indicates a significant difference $(\mathrm{P}<0.05)$.

SI of a marker, i.e., non- or almost non-marker expressing and higher marker expressing tumours. The resulting pairs of a further marker were subsequently assessed for differences. A cut-off value of $\leq 2$ was taken to distinguish between non- or almost non-marker expressing and higher marker expressing tumours. This cut-off was used in all subsequent analyses.

\section{Results}

Tumour characteristics. Patient and tumour characteristics are summarised in Table I.

Immunohistochemistry and phenotype assessment. Paraffin sections of tumours were immunostained against CEA to visualize the malignant cells, against CD45 to detect infiltrating leucocytes, and against HLA-DR $\alpha$ chain and HLA-DR for

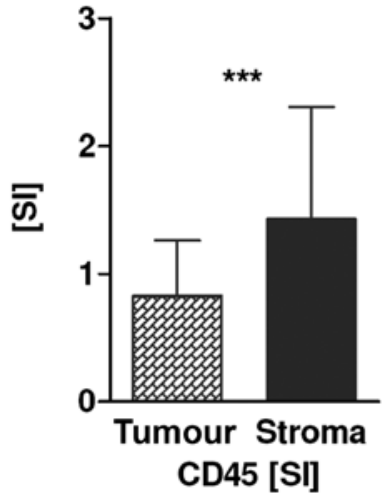

Figure 3. Comparison of leucocyte infiltration of tumour and stromal tissue according to the CD45 SI. Significantly fewer leucocytes have been intermingled with the tumour cells than with the stromal tissue. ${ }^{* * * *}$ Indicates a highly significant difference $(\mathrm{P}<0.0001)$.

MHC class II, as well as HLA-A and $\beta 2$ microglobulin for MHC class I (Fig. 1). There was no CEA expression in $3.7 \%$ of the tumours and $2.0 \%$ of tumours did not express any detectable markers of MHC class I or II. A significant albeit small difference could be proven for tumour grade and expression of CEA indicating that tumours with higher grade and with lower differentiation expressed less CEA (Fig. 2).

MHC class I and II marker expression in tumours and stroma. Expression of MHC related markers was compared between tumour cells and stromal tissue. The only difference between tumourous tissue and stromal tissue was found for CD45. Tumourous tissue exhibited a lower SI and herewith lower content of intermingled leucocytes than the surrounding stroma $(\mathrm{P}<0.0001$, Fig. 3). No differences in the relative 


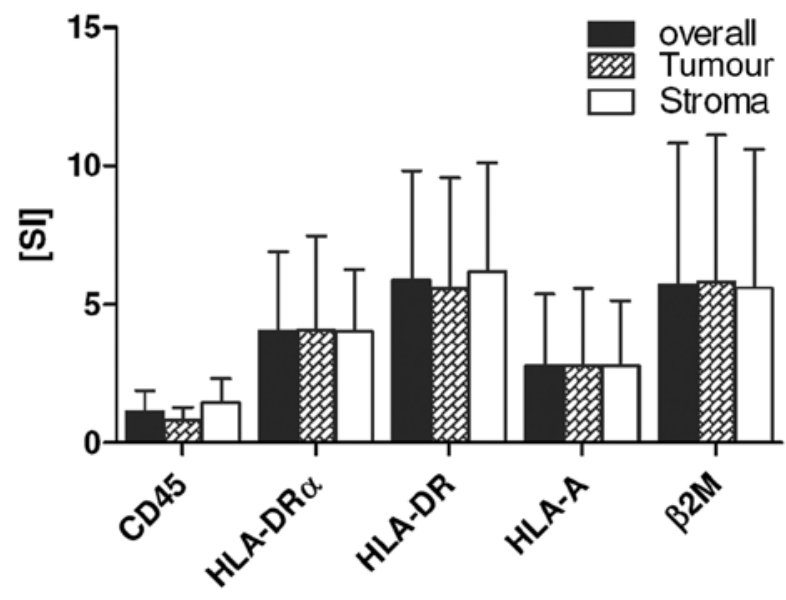

Figure 4. Comparison of overall, tumoural and stromal expression according to SI for MHC class I and II markers.

expression between tumour and stromal tissue were found for the markers HLA-DR $\alpha$ chain, HLA-DR, HLA-A and $\beta 2$ microglobulin (Fig. 4). However, HLA-A expression was not detectable in $31 \%$ of the tumours and was low in another $38 \%$ of the tumours.

On the contrary, the comparison of SI between tumourous and stromal tissue revealed a significant correlation after grouping according to lower and higher SI, in that the low $\mathrm{SI}$ in the stromal tissue correlated with the low expression in the tumourous tissue (and vice versa) for the MHC class II markers HLA-DR $\alpha$ chain $(\mathrm{P}<0.002)$ and HLA-DR $(\mathrm{P}<0.0001)$ and for the MHC class I markers HLA-A $(\mathrm{P}<0.0001)$ and $\beta 2$ microglobulin ( $\mathrm{P}<0.0001$, Fig. 5).

Inverse expression of MHC class I and II markers. The MHC class II marker HLA-DR was not uniformly expressed in tumourous tissues. A low expression in the tumour correlated with a low expression of HLA-DR in the stroma. However, the lower SI of the MHC class II marker HLA-DR in the tumour correlated with an inversely higher stromal SI of the MHC class I marker HLA-A ( $\mathrm{P}=0.03)$ and $\beta 2$ microglobulin and vice versa. A similar inverse staining pattern could be observed, when tumours were grouped according to a low vs. high staining index of stromal HLA-DR. A low stromal expression of this MHC class II marker correlated with a higher expression of the MHC class I markers HLA-A $(\mathrm{P}=0.02)$ and $\beta 2$ microglobulin $(\mathrm{P}=0.06)$ in the stroma, but also in the tumours. The expression of the MHC class II marker HLA-DR $\alpha$ chain was similar to HLA-DR in that a low expression in the tumour correlated with a low expression of HLA-DR in the stroma. The expression pattern did not reach significance when compared with any of the other markers (data not shown).

The expression of the MHC class I marker HLA-A in tumour cells significantly correlated with $\beta 2$ microglobulin, i.e., a lower HLA-A expression was accompanied by a lower $\beta 2$ microglobulin expression $(\mathrm{P}=0.0003)$. In the group with lower tumour SI for HLA-A there was a inversely higher stromal HLA-DR SI $(\mathrm{P}=0.007)$ as in tissues with higher HLA-A SI, while there was a positive correlation with the stromal MHC class I markers HLA-A $(\mathrm{P}=0.0001)$ and $\beta 2$ microglobulin $(\mathrm{P}=0.0002)$. Grouping for low vs. high stromal HLA-A SI

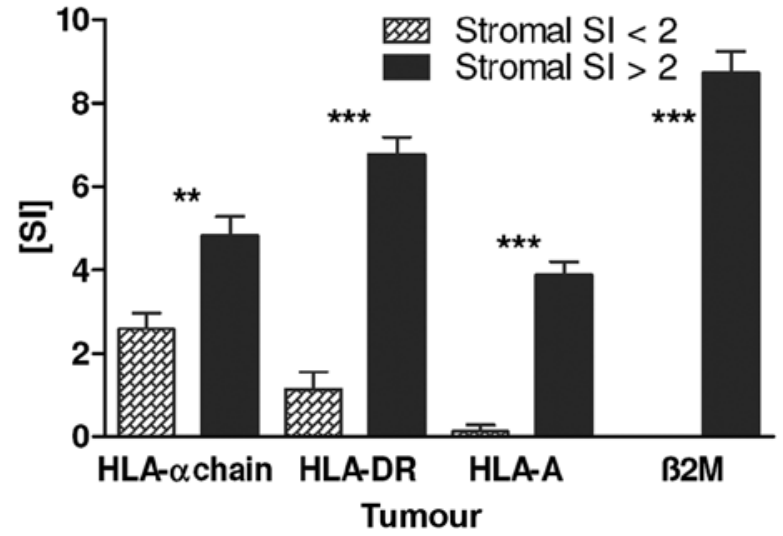

Figure 5. Comparison of SI for MHC class I and II markers. A low marker expression according to SI in the stromal tissue correlated with the low expression in the tumourous tissue (and vice versa). ${ }^{* *}$, and ${ }^{* * * *}$ indicate highly significant differences $(\mathrm{P}<0.002$ and $\mathrm{P}<0.0001$, respectively).

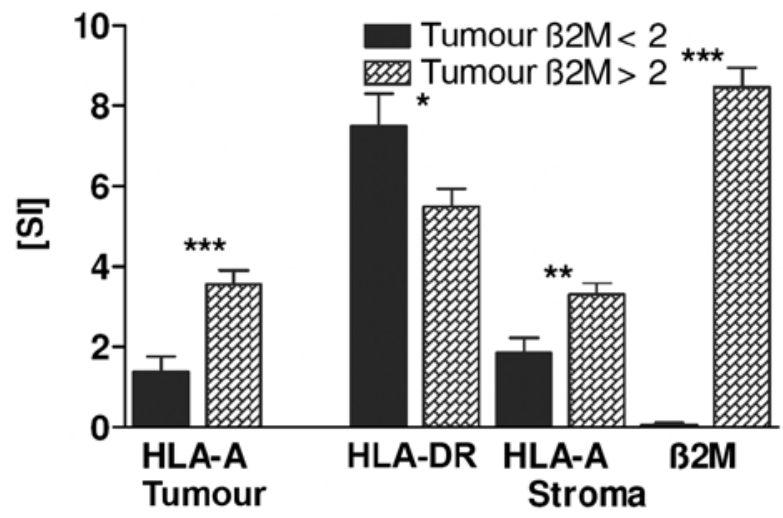

Figure 6. Comparison of SI for $\beta 2$ microglobulin and MHC class I and II markers. A low marker expression according to SI in the tumour tissue correlated with the low expression of HLA-A in the tumourous tissue and in the stroma but revealed an inverse expression pattern for HLA-DR (and vice versa). ${ }^{*},{ }^{* *}$, and ${ }^{* * *}$ indicate (highly) significant differences $(\mathrm{P}=0.018$, $\mathrm{P}=0.0037$, and $\mathrm{P}<0.0002$, respectively).

demonstrated, that a low expression in the stroma correlated with a low expression of tumour HLA-A, stromal $(\mathrm{P}=0.005)$ and tumour $\beta 2$ microglobulin $(\mathrm{P}=0.002)$ and with an inversely higher stromal HLA-DR $(\mathrm{P}=0.04)$.

Grouping for low vs. high tumour $\beta 2$ microglobulin SI showed that a low expression in the tumours correlated with a low HLA-A expression within the tumour $(\mathrm{P}=0.0002)$ but also a low expression of $\beta 2$ microglobulin $(\mathrm{P}<0.0001)$ and HLA-A in the stroma $(\mathrm{P}=0.0037)$, which was accompanied by an inversely higher HLA-DR expression in the stroma $(\mathrm{P}=0.018$, Fig. 6).

Determination of HLA-genotype. Low resolution HLA-genotyping of genomic DNA was possible in 49 cases for DRB1* and in 48 cases for DQB1 ${ }^{*}$. Results are depicted in Table II and Figs. 7 and 8 . The P-values of the $\mathrm{Z}_{\max }$ test were 0.328 for $\mathrm{DRB1}^{*}$ and 0.176 for DQB1*. 


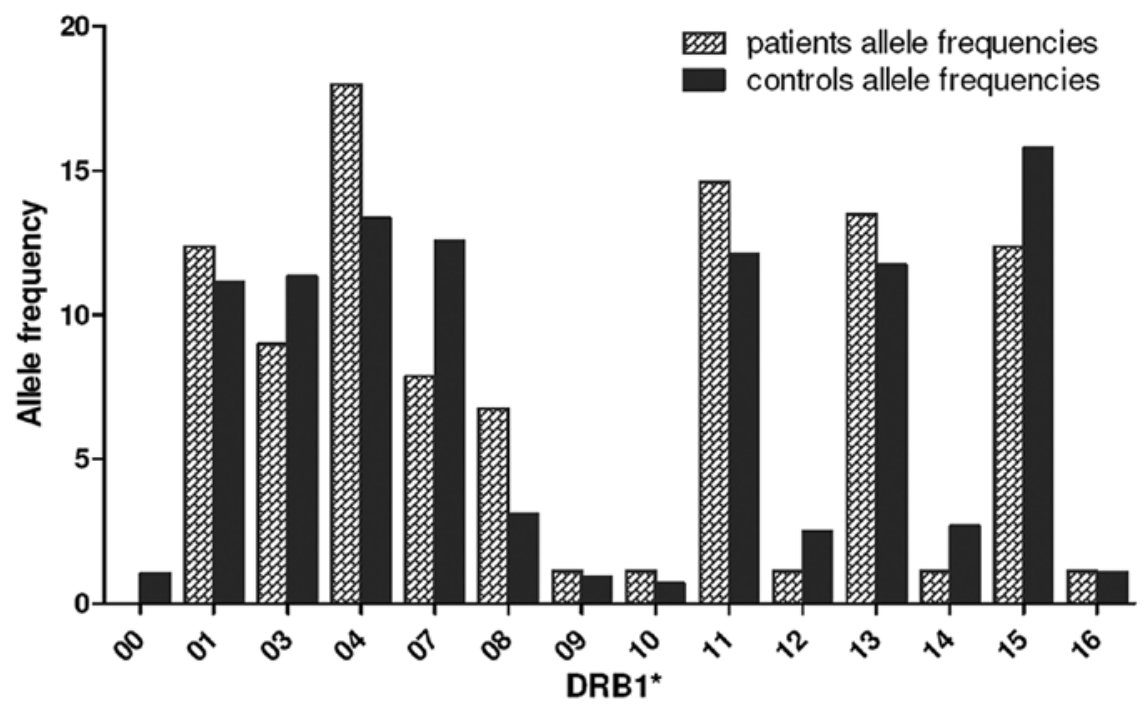

Figure 7. Allele frequencies of patients with colorectal cancer in comparison with HLA gene frequencies of normal bone marrow donors for the DRB1 locus as reported by Mueller et al (44). The P-values of $\mathrm{Z}_{\max }$ test was 0.328 .

Table II. Allele frequencies of patients with colorectal cancer are depicted in comparison with HLA gene frequencies of normal bone marrow donors (44).

\begin{tabular}{cccc}
\hline & $\begin{array}{c}\text { Patient allele } \\
\text { counts }\end{array}$ & $\begin{array}{c}\text { Patient allele } \\
\text { frequencies }\end{array}$ & $\begin{array}{c}\text { Controls allele } \\
\text { frequencies }\end{array}$ \\
\hline DRB1* & & & \\
00 & 0 & 0.00 & 1.03 \\
01 & 11 & 12.36 & 11.16 \\
03 & 8 & 8.99 & 11.33 \\
04 & 16 & 17.98 & 13.36 \\
07 & 7 & 7.87 & 12.56 \\
08 & 6 & 6.74 & 3.09 \\
09 & 1 & 1.12 & 0.91 \\
10 & 1 & 1.12 & 0.70 \\
11 & 13 & 14.61 & 12.11 \\
12 & 1 & 1.12 & 2.49 \\
13 & 12 & 13.48 & 11.75 \\
14 & 1 & 1.12 & 2.67 \\
15 & 11 & 12.36 & 15.77 \\
16 & 1 & 1.12 & 1.08 \\
All & 89 & 100 & 100 \\
DQB1 & & & \\
02 & 16 & 19.51 & 24.31 \\
03 & 28 & 34.15 & 37.67 \\
04 & 6 & 7.32 & 3.57 \\
05 & 13 & 15.85 & 15.96 \\
06 & 19 & 23.17 & 18.49 \\
All & 82 & 100 & 100 \\
\hline & & & \\
\hline
\end{tabular}

\section{Discussion}

In the study presented, we sought to analyse immunological features of MHC class I and II as a possible prerequisite of

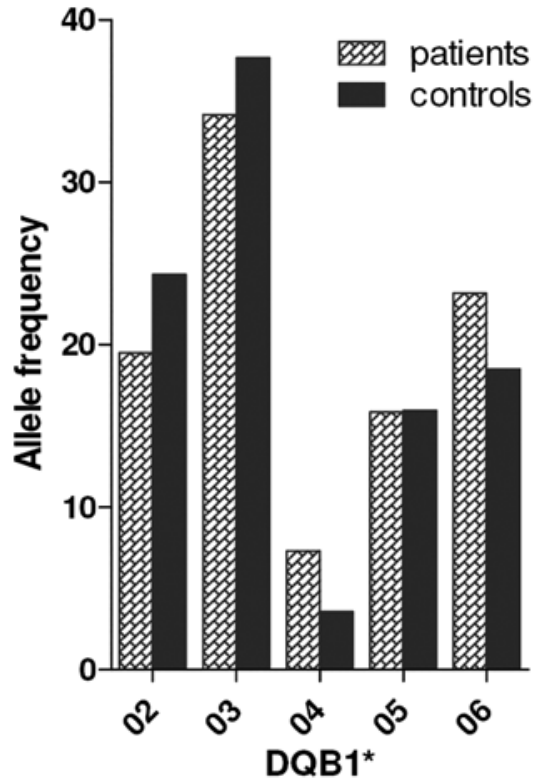

Figure 8. Allele frequencies of patients with colorectal cancer in comparison with HLA gene frequencies of normal bone marrow donors for the DQB1 locus (44). The P-values of $Z_{\max }$ test is 0.176 .

inflammation and inflammatory response and eventually neoplastic degeneration in sporadic colorectal carcinoma. We found a wide inter- and intra-tumour variation in the proportional expression of MHC class I and II markers and of leucocyte infiltration according to common leucocyte antigen expression.

The immunohistochemical analyses suggests a similar expression level of the investigated markers in tumours and stromal tissue. This finding is in accordance with former data on microdissection (45). However, microarray data are not unequivocal and, hence, still a matter of debate. The similar expression patterns of the investigated markers in tumours and stromal tissue might characterise the microenvironment in a given tumour as a 'functional unit' of stromal and tumourous tissue with respect to the immunological behaviour. However, 
completely different expression patterns have been reported for metabolic features in tumour and stromal cells, which also let investigators to hypothesise of a functional unit of the highly metabolising and growing tumour cells and their fostering stromal bystander cells (46).

Contrary to former reports we found a correlation between $\beta 2$ microglobulin and the MHC class I marker expression (47). In the normal cell $\beta 2$ microglobulin associates with the $\alpha$ chain of MHC class I molecules and with class I-like molecules such as CD1 or Qa. This association is necessary for the cell surface expression of MHC class I and the stability of the peptide binding groove. Indeed, if $\beta 2$ microglobulin is lacking, only very limited amounts of MHC class I molecules can be detected on the cell surface as has been shown in $\beta 2$ microglobulin knockout mice. In the absence of MHC class I, development CD8 T cells development is hampered which results in an impairment of acquired immunity. In fact, $\beta 2$ microglobulin knockout mice have been used in cell transplant studies because of the excellent engraftment in these immune-deficient hosts $(48,49)$. This could translate into a diminished immunological response to a developing tumour and its antigens.

The expression of HLA-A and $\beta 2$ microglobulin as markers and surrogate markers for MHC class I was in general low in our study. HLA-A expression was not detectable in $31 \%$ of tumours and was low in another $38 \%$ of tumours. Similar results have been reported by others (50) and attributed to a possible mechanism of tumour escape from immune surveillance as discussed above. Although this concept is intriguing and seeks to explain an uncontrolled tumour growth in spite of an apparent normal immune system this concept does not provide an explanation as to why an immune reaction against the own cells, even if tumourous, should exist. Still, it may be possible, that tumour and stromal tissue do behave similarly but differently in terms of their immunological responses compared to other tissues not affected by tumour growth.

Our results show an inverse expression of I and II markers which cannot be easily explained. The principal function of MHC class II molecules includes the processing and subsequent presentation of external antigens to immunocompetent T-helper cells. This involvement of MHC class II, the TCR complex and CD4 molecules in the control and regulation of T-helper lymphocytes is well understood $(51,52)$. The presentation of external antigens via MHC class II can induce either stimulation of immunological response or its blunting via apoptosis $(53,54)$. However, it was noted that MHC class II molecules not only present external antigens but can also present cytosolic and nuclear antigens. This process involves macroautophagy and chaperone-mediated autophagy $(55,56)$. It could result in cross-presentation of viral antigens as well as of tumour-related or even self antigens. Indeed, analyses of the MHC class II ligandome demonstrated the presentation of up to $30 \%$ self antigens (57). The implication with respect to the tumour is as yet to be determined, but could offer further explanation for the possible immune escape of colorectal cancer. Of note, macroautophagy-related MHC class II presentation of MUC1-derived peptides by dendritic cells significantly inhibited MUC1-specific CD4 cell proliferation (58). As to whether an expression of MHC class II molecules could also be a hint for the involvement of an infectious trigger, cannot be concluded from the data presented. Also, the infection with a specific pathogen could trigger the expression of MHC class II molecules. Moreover, it is intriguing to hypothesise a situation similar to that demonstrated in cervix carcinoma subsequent to the infection with the human papillomavirus. In the cervix carcinoma a specific HLA type enhances the infection with the human papillomavirus. Therefore, we sought to identify the HLA type of the MHC class II locus by low resolution HLA-genotyping. However, although some differences can be seen, a single prevailing HLA type could not be determined due to the small number of cases. Therefore, we fail to demonstrate a link between the HLA system and the sporadic colorectal carcinoma.

In summary we found a similar immunological marker expression in tumour and stromal tissue, which we interpret as a similar functional difference with respect to the immune system or immunogenicity. Second, we confirmed an absent or decreased expression of MHC class I markers in sporadic colon carcinoma. This lacking MHC class I expression results in the loss or at least decreased antigen presentation. Therefore, a blunted immunological response can be hypothesised. Third, we found an inverse expression of MHC class I and II markers. To date it is not clear as to whether this constitutes a true hint of the involvement of an external pathogen in the induction of the process of transformation. Further studies on MHC class I and II genotype and phenotype relation in colorectal cancer are necessary and may help to identify triggering mechanisms of tumourigenesis, involved markers and possible subsequent immune escape.

\section{Acknowledgements}

We wish to thank H. Zimmermann-Höffken for careful assistance during the laboratory experiments.

\section{References}

1. O'Brien MJ and Gibbons D: The adenoma-carcinoma sequence in colorectal neoplasia. Surg Oncol Clin N Am 5: 513-530, 1996.

2. Larsson SC, Orsini N and Wolk A: Diabetes mellitus and risk of colorectal cancer: a meta-analysis. J Natl Cancer Inst 97: 1679-1687, 2005.

3. Wei EK, Giovannucci E, Fuchs CS, Willett WC and Mantzoros CS: Low plasma adiponectin levels and risk of colorectal cancer in men: a prospective study. J Natl Cancer Inst 97: 1688-1694, 2005.

4. Cairns SR, Scholefield JH, Steele RJ, et al: Guidelines for colorectal cancer screening and surveillance in moderate and high risk groups (update from 2002). Gut 59: 666-689, 2010.

5. Stallmach A, Bielecki $C$ and Schmidt C: Malignant transformation in inflammatory bowel disease - surveillance guide. Dig Dis 27: 584-590, 2009.

6. Triantafillidis JK, Nasioulas G and Kosmidis PA: Colorectal cancer and inflammatory bowel disease: epidemiology, risk factors, mechanisms of carcinogenesis and prevention strategies. Anticancer Res 29: 2727-2737, 2009.

7. Thompson AI and Lees CW: Genetics of ulcerative colitis Inflamm Bowel Dis 17: 831-848, 2011.

8. Cooney R and Jewell D: The genetic basis of inflammatory bowel disease. Dig Dis 27: 428-442, 2009.

9. Cooney R, Baker J, Brain O, et al: NOD2 stimulation induces autophagy in dendritic cells influencing bacterial handling and antigen presentation. Nat Med 16: 90-97, 2010.

10. Travassos LH, Carneiro LA, Ramjeet M, et al: Nod1 and Nod2 direct autophagy by recruiting ATG16L1 to the plasma membrane at the site of bacterial entry. Nat Immunol 11: 55-62, 2010.

11. Schetter AJ, Nguyen GH, Bowman ED, et al: Association of inflammation-related and microRNA gene expression with cancerspecific mortality of colon adenocarcinoma. Clin Cancer Res 15: 5878-5887, 2009 . 
12. Hojjat-Farsangi M, Jeddi-Tehrani M, Amirzargar AA, et al: Human leukocyte antigen class II allele association to disease progression in Iranian patients with chronic lymphocytic leukemia. Hum Immunol 69: 666-674, 2008.

13. De Petris L, Bergfeldt K, Hising C, et al: Correlation between HLA-A2 gene frequency, latitude, ovarian and prostate cancer mortality rates. Med Oncol 21: 49-52, 2004.

14. Diepstra A, Niens M, te Meerman GJ, Poppema S and van den Berg A: Genetic susceptibility to Hodgkin's lymphoma associated with the human leukocyte antigen region. Eur J Haematol Suppl 66: 34-41, 2005.

15. Staratschek-Jox A, Shugart YY, Strom SS, Nagler A and Taylor GM: Genetic susceptibility to Hodgkin's lymphoma and to secondary cancer: workshop report. Ann Oncol 13 (Suppl 1): S30-S33, 2002

16. Harty LC, Lin AY, Goldstein AM, et al: HLA-DR, HLA-DQ, and TAP genes in familial Hodgkin disease. Blood 99: 690-693, 2002.

17. Kageshita T, Naruse T, Hirai S, et al: Molecular genetic analysis of HLA class II alleles in Japanese patients with melanoma. Tissue Antigens 49: 466-470, 1997.

18. Watanabe Y, Aoyama N, Sakai T, et al: HLA-DQB1 locus and gastric cancer in Helicobacter pylori infection. $\mathrm{J}$ Gastroenterol Hepatol 21: 420-424, 2006.

19. Glew SS, Duggan-Keen M, Cabrera T and Stern PL: HLA class II antigen expression in human papillomavirus-associated cervical cancer. Cancer Res 52: 4009-4016, 1992.

20. Mehal WZ, Lo YM, Herrington CS, et al: Role of human papillomavirus in determining the HLA associated risk of cervical carcinogenesis. J Clin Pathol 47: 1077-1081, 1994.

21. Nawa A, Nishiyama Y, Kobayashi T, et al: Association of human leukocyte antigen-B1*03 with cervical cancer in Japanese women aged 35 years and younger. Cancer 75: 518-521, 1995.

22. Eiguchi K, Tatti S, Alonio LV, et al: Association of DRB1 and DQB1 HLA class II polymorphisms in high-grade and neoplastic cervical lesions of women from Argentina. J Low Genit Tract Dis 12: 262-268, 2008

23. Li PK, Leung NW, Poon AS, Wong KC, Chan TH and Lai KN: Molecular genetics of major histocompatibility complex class II genes in hepatocellular carcinoma. Dig Dis Sci 40: 1542-1546, 1995.

24. De Re V, Caggiari L, Simula MP, et al: Role of the HLA class II: HCV-related disorders. Ann NY Acad Sci 1107: 308-318, 2007.

25. Ando T, Ishikawa T, Kato H, et al: Synergistic effect of HLA class II loci and cytokine gene polymorphisms on the risk of gastric cancer in Japanese patients with Helicobacter pylori infection. Int J Cancer 125: 2595-2602, 2009.

26. Magnusson PKE, Enroth H, Eriksson I, et al: Gastric cancer and human leukocyte antigen: distinct DQ and DR alleles are associated with development of gastric cancer and infection by Helicobacter pylori. Cancer Res 61: 2684-2689, 2001.

27. Juhasz F, Kozma L, Stenszky V, Gyory F, Luckas G and Farid NR Well differentiated thyroid carcinoma is associated with human lymphocyte antigen D-related 11 in Eastern Hungarians: a case of changing circumstances. Cancer 104: 1603-1608, 2005

28. Rigopoulou D, Martinez-Laso J, Martinez-Tello F, et al: Both class I and class II HLA antigens are thyroid cancer susceptibility factors. Tissue Antigens 43: 281-285, 1994.

29. Mottironi VD, Banks SM, Pollara B and Rudofsky UH: HLA and survival in lung cancer. Clin Immunol Immunopathol 45: 55-62, 1987.

30. Markman M, Braine HG and Abeloff MD: Histocompatibility antigens in small cell carcinoma of the lung. Cancer 54: 2943-2945, 1984.

31. Tokumoto H: Analysis of HLA-DRB1-related alleles in Japanese patients with lung cancer - relationship to genetic susceptibility and resistance to lung cancer. J Cancer Res Clin Oncol 124 511-516, 1998.

32. Naugler C and Liwski R: HLA risk markers for chronic myelogenous leukemia in Eastern Canada. Leuk Lymphoma 50: 254-259, 2009.

33. Manns A, Hanchard B, Morgan OS, et al: Human leukocyte antigen class II alleles associated with human T-cell lymphotropic virus type I infection and adult T-cell leukemia/lymphoma in a Black population. J Natl Cancer Inst 90: 617-622, 1998.

34. Fuggle SV, Kirkley J, Ting A and Morris PJ: A monoclonal antibody recognizing HLA-DR2 on malignant and activated cells. Immunogenetics 18: 415-428, 1983.

35. Nunez-Roldan A, Szer I, Toguchi T, Cuttner J and Winchester R: Association of certain Ia allotypes with the occurrence of chronic lymphocytic leukemia. Recognition by a monoclonal anti-Ia reagent of a susceptibility determinant not in the DR series. J Exp Med 156: 1872-1877, 1982.
36. Beskow AH, Josefsson AM and Gyllensten UB: HLA class II alleles associated with infection by HPV16 in cervical cancer in situ. Int J Cancer 93: 817-822, 2001.

37. Neuman RJ, Huettner PC, Li L, et al: Association between DQB1 and cervical cancer in patients with human papillomavirus and family controls. Obstet Gynecol 95: 134-140, 2000.

38. Moller P, Momburg F, Koretz K, et al: Influence of major histocompatibility complex class I and II antigens on survival in colorectal carcinoma. Cancer Res 51: 729-736, 1991.

39. Speetjens FM, de Bruin EC, Morreau H, et al: Clinical impact of HLA class I expression in rectal cancer. Cancer Immunol Immunother 57: 601-609, 2008.

40. Benevolo M, Mottolese M, Piperno G, et al: HLA-A, -B, -C expression in colon carcinoma mimics that of the normal colonic mucosa and is prognostically relevant. Am J Surg Pathol 31: 76-84, 2007.

41. Eyal A, Levin I, Segal S, Levi I, Klein B and Kuperman O: Variation of HLA-ABC surface antigen expression on adenocarcinoma of the colon in correlation with the degree of differentiation. Nat Immun Cell Growth Regul 9: 222-227, 1990.

42. Norazmi M, Hohmann AW, Skinner JM and Bradley J: Expression of MHC class I and class II antigens in colonic carcinomas. Pathology 21: 248-253, 1989.

43. DNA Preparation from Paraffin Tissue. In: http://www.riedlab. nci.nih.gov/protocols.asp.

44. Mueller CR, Goldmann SF and Wegener GS: Distribution of tissue antigens in the German normal population. In: HLA 1998. Gjerston DW and Terasaki PT (eds). The American Society for Histocompatibility and Immunogenetics, Lenexa, pp154-157, 1998.

45. Staub E, Groene J, Heinze M, et al: Genome-wide expression patterns of invasion front, inner tumor mass and surrounding normal epithelium of colorectal tumors. Mol Cancer 6: 79, 2007.

46. Koukourakis MI, Giatromanolaki A, Harris AL and Sivridis E: Comparison of metabolic pathways between cancer cells and stromal cells in colorectal carcinomas: a metabolic survival role for tumor-associated stroma. Cancer Res 66: 632-637, 2006

47. Durrant LG, Ballantyne KC, Armitage NC, et al: Quantitation of MHC antigen expression on colorectal tumours and its association with tumour progression. Br J Cancer 56: 425-432, 1987.

48. Ito M, Hiramatsu H, Kobayashi K, et al: NOD/SCID/gamma(c) (null) mouse: an excellent recipient mouse model for engraftment of human cells. Blood 100: 3175-3182, 2002.

49. Somigliana E, Vigano P, Filardo P, Candiani M, Vignali M and Panina-Bordignon P: Use of knockout transgenic mice in the study of endometriosis: insights from mice lacking beta(2)microglobulin and interleukin-12p40. Fertil Steril 75: 203-206, 2001.

50. Garrido F and Ruiz-Cabello F: MHC expression on human tumors - its relevance for local tumor growth and metastasis. Semin Cancer Biol 2: 3-10, 1991.

51. Konig R, Shen X, Maroto R and Denning TL: The role of CD4 in regulating homeostasis of $\mathrm{T}$ helper cells. Immunol Res 25: 115-130, 2002

52. Maroto R, Shen X and Konig R: Requirement for efficient interactions between CD4 and MHC class II molecules for survival of resting $\mathrm{CD}^{+} \mathrm{T}$ lymphocytes in vivo and for activation-induced cell death. J Immunol 162: 5973-5980, 1999.

53. Boehme SA, Zheng L and Lenardo MJ: Analysis of the CD4 coreceptor and activation-induced costimulatory molecules in antigen-mediated mature T lymphocyte death. J Immunol 155: 1703-1712, 1995

54. Riberdy JM, Mostaghel E and Doyle C: Disruption of the CD4-major histocompatibility complex class II interaction blocks the development of CD4(+) T cells in vivo. Proc Natl Acad Sci USA 95: 4493-4498, 1998.

55. Gannage M and Munz C: MHC presentation via autophagy and how viruses escape from it. Semin Immunopathol 32: 373-381, 2010.

56. Gannage M and Munz C: Autophagy in MHC class II presentation of endogenous antigens. Curr Top Microbiol Immunol 335: 123-140, 2009

57. Deng jel J, Schoor O, Fischer R, et al: Autophagy promotes MHC class II presentation of peptides from intracellular source proteins. Proc Natl Acad Sci USA 102: 7922-7927, 2005.

58. Dorfel D, Appel S, Grunebach F, et al: Processing and presentation of HLA class I and II epitopes by dendritic cells after transfection with in vitro-transcribed MUC1 RNA. Blood 105: 3199-3205, 2005 